\title{
La problemática de adicciones en pueblos indígenas de Colombia y la convención sobre los derechos de las personas con discapacidad*
}

\author{
Angélica María Gutiérrez Ramos**
}

\begin{abstract}
RESUMEN
El incremento del consumo de sustancias psicoactivas entre los pueblos indígenas de Colombia es un problema en ascenso. El ordenamiento jurídico colombiano ha reconocido la adicción como enfermedad mental, ha elevado el derecho a la salud mental a la categoría de derecho fundamental, y ha incorporado normas para garantizar el derecho a la salud de los pueblos indígenas. Sin embargo, todavía existe en esta materia una brecha entre las normas y su aplicación. Este artículo sostiene que una de las principales razones detrás de esa desconexión entre las normas y la realidad está ligada a las limitaciones de los modelos acerca de la naturaleza de la adicción que han permeado el ordenamiento colombiano, y argumenta que la Convención sobre los Derechos de las Personas con Discapacidad es el instrumento normativo que permite comprender mejor el fenómeno de las adicciones. Esto por cuanto dicho instrumento permite ver la adicción como el resultado de vulnerabilidades socialmente causadas, que confluyen para socavar-mas no extinguir- la autonomía del individuo. Por esta razón, analizar la adicción desde la óptica de este tratado, es el primer paso para acuñar soluciones encaminadas a combatir esas vulnerabilidades, y brindarle tratamiento al adicto sin negarle su carácter de agente moral.
\end{abstract}

Adicciones en pueblos indígenas - salud mental - discapacidad

\section{The issue of addiction in Colombia's indigenous people and the un convention on the rights of persons with disabilities}

\begin{abstract}
The increase in substance use among Colombian indigenous people is currently a public bealth issue. Colombian Law has recognized that addiction is a mental health disorder, it has elevated mental health to the category of a fundamental right and has incorporated multiple norms to ensure the right to health for indigenous people. However, there is still in this regard a disconnect between what the law states and how it is being implemented. This article holds that one of the
\end{abstract}

* Este artículo toca algunos de los puntos que abordé en mi tesis de maestría y profundiza en ellos.

** Abogada, Facultad de Jurisprudencia, Universidad del Rosario, Colombia. Máster en Salud Mental, Ética y Derecho, King's College London, Reino Unido. Profesora de Introducción al Derecho y Derecho de Familia, Facultad de Jurisprudencia, Universidad del Rosario, Colombia. Correo electrónico: angelicam. gutierrez@urosario.edu.co

Artículo recibido el 22.2.2020 y aceptado para su publicación el 7.7.2020. 
main reasons behind this disconnect is associated to the limitations of the models explaining the nature of addiction that have permeated Colombian Law. It also argues that the Convention on the Rights of Persons with Disabilities is the legal norm which enables the best understanding of the phenomenon of addiction, since it allows to see addiction as the result of a series of socially-caused vulnerabilities that erode -but do not extinguish-autonomy, and as such it fosters the adoption of solutions that seek the elimination of those vulnerabilities and the implementation of treatments that do not deny the addicts' agency.

Addictions in indigenous people - mental health - disability

\section{INTRODUCCIÓN}

$\mathrm{V}$ arios estudios han demostrado que hay una mayor prevalencia del consumo de sustancias psicoactivas ${ }^{1}$ entre los pueblos indígenas que entre la población general $^{2}$. Este fenómeno se ha atribuido al trauma histórico que han padecido estos pueblos debido a la opresión colonial, la discriminación y la pérdida de territorio; trauma que ha contribuido al deterioro de su salud mental y ha fomentado el abuso de sustancias psicoactivas ${ }^{3}$. Esta situación no le es ajena a Colombia, donde la historia de opresión y violencia hacia la población indígena ha impactado negativamente en su salud mental.

El ordenamiento jurídico colombiano, que antes propendía por la penalización del consumo de estas sustancias, ha calificado la adicción como enfermedad mental y ha elevado el derecho a la salud mental a la categoría de derecho fundamental. Dicho ordenamiento ha incorporado además distintas normas tendientes a garantizar el derecho a la salud de los indígenas. Sin embargo, pese a esos esfuerzos normativos, la situación de adicciones en los pueblos indígenas de Colombia es apremiante; la brecha entre el contenido de las normas y su aplicación práctica es evidente. Este artículo sostiene que una de las principales razones detrás de esa brecha está asociada a las limitaciones de los modelos acuñados para explicar la naturaleza de la adicción, y arguye que de los instrumentos normativos relevantes en esta materia, es la Convención sobre los Derechos de las Personas con Discapacidad (CDPD) el que permite comprender mejor el fenómeno de las adicciones.

Para ese efecto la argumentación se dividirá en cinco acápites: en el primero haremos una breve exposición acerca del fenómeno de las adicciones en los pueblos indígenas de Colombia. En segundo lugar, presentaremos el panorama normativo que resulta relevante en este contexto, para concluir que pese a la profusa normatividad que

${ }^{1}$ Las sustancias psicoactivas a las que hace referencia este artículo no son las sustancias tradicionales, sino las sustancias comunes ajenas a la cosmovisión ancestral de los pueblos indígenas.

${ }^{2}$ Universidad de Antioquia y Organización Indígena de Antioquia, 2006, p. 132, French, 2004, p. 81 y SZLEMKO et al., 2006, p. 435.

${ }^{3}$ Universidad de Antioquia y Organización Indígena de Antioquia, 2006, pp. 132-133. 
respecto del particular existe, ella tiene pocas repercusiones prácticas. En tercer lugar, presentaremos los dos principales modelos que se han acuñado para explicar la naturaleza de la adicción -y que han tenido un claro impacto en nuestras normas jurídicas-, para concluir que ambos se quedan cortos en la consecución del propósito que persiguen. En cuarto lugar, expondremos la alternativa conceptual que consideramos más adecuada para entender la adicción y que, de ser acogida, permitiría avanzar en la consecución de soluciones para la situación de adicciones en pueblos indígenas de Colombia. Y por último, argüiremos que, de las normas mencionadas en el acápite respecto del panorama normativo, la CDPD es el instrumento que más se acopla a la manera de entender la adicción expuesta en este artículo.

\section{LAS Adicciones EN los Pueblos indíGenAs de Colombia}

Estudios de salud mental en los pueblos indígenas de las Américas han encontrado que el alcoholismo es una de las enfermedades crónicas más prevalentes en esa población ${ }^{4}$. La evidencia apunta a la existencia de un elevado patrón de consumo de alcohol y sustancias psicoactivas entre los pueblos indígenas, superior al patrón de consumo de la población general ${ }^{5}$. Ante esta alarmante realidad es apenas lógico preguntarse por las causas que han motivado este fenómeno. Un sector de la literatura ha identificado como posibles precursores de este estado de cosas, el trauma histórico que durante siglos han padecido los pueblos indígenas a causa de las políticas coloniales, la pérdida de territorio y los procesos de aculturación ${ }^{6}$. Estas circunstancias se han convertido en el caldo de cultivo de diversos trastornos y problemas de salud mental como la depresión, el suicidio y el abuso de sustancias ${ }^{7}$.

En consonancia con lo anterior, la Organización Panamericana de la Salud ha afirmado que distintos factores como el influjo del pasado colonial, los altos niveles de pobreza y el acceso limitado a servicios de educación y salud, hacen que la población indígena de las Américas sea especialmente vulnerable al consumo excesivo de alco$\mathrm{hol}^{8}$. Infortunadamente, se ha demostrado que ciertos trastornos psiquiátricos son más comunes en grupos sociales más desventajados, y no es poco usual que, ante el racismo institucional, ciertas minorías étnicas -como los indígenas- estén en los niveles socioeconómicos más bajos y, en consecuencia, tengan una salud mental deficiente 9 . Ante

\footnotetext{
${ }^{4}$ Universidad de Antioquia y Organización Indígena de Antioquia, 2006, p. 60 y Szlemko et al., 2006, p. 435.

${ }^{5}$ Universidad de Antioquia y Organización Indígena de Antioquia, 2006, p. 132 y French, 2004 , p. 81.

${ }^{6}$ Universidad de Antioquia y Organización Indígena de Antioquia, 2006, pp. 132-133 y WeSLEy-EsQuimaUX y SMOLEWSKI, 2004, p. 64.

${ }^{7}$ Universidad de Antioquia y Organización Indígena de Antioquia, 2006, pp. 132-133.

${ }^{8}$ Organización Panamericana de la Salud, 2011, p. 1.

${ }^{9}$ McGeorge y Bhugra, 2013, pp. 8-9.
} 
esta realidad, la prevalencia del consumo elevado de sustancias psicoactivas en pueblos indígenas resulta todo menos sorprendente.

Esta situación no es extraña para Colombia, donde los indígenas no solamente están expuestos a los factores ya referidos -influjo del pasado colonial, altos niveles de pobreza y acceso limitado a servicios-, sino que además han tenido que vivir en carne propia la pérdida de identidad cultural y el desarraigo ocasionados por las cruentas luchas entre los actores armados ilegales que, motivados por el lucrativo negocio del narcotráfico, se han enfrentado durante décadas por el control territorial y han desplazado a los indígenas de sus territorios ${ }^{10}$. Esto resulta especialmente problemático debido al vínculo estrecho que existe entre la salud mental de las comunidades indígenas y la posibilidad que ellas tienen de habitar sus territorios ${ }^{11}$; vínculo que ha sido reconocido por el Comité de Derechos Económicos, Sociales y Culturales (CDESC) al señalar el papel que tienen las tierras de los pueblos indígenas en su salud ${ }^{12}$.

Todos estos factores han contribuido al deterioro de la salud mental de los indígenas en Colombia. No es fortuito que la Organización Nacional Indígena de Colombia (ONIC) haya manifestado su preocupación por el incremento en el suicidio y el uso de sustancias en sus comunidades ${ }^{13}$. Varios pueblos indígenas en los departamentos de Chocó, Cauca y Vaupés, entre otros, están presentando un alza en las tasas de suicido ${ }^{14}$. Las autoridades del Vaupés han establecido que la mayoría de casos de suicidio en ese departamento están asociados al consumo de alcohol y drogas ${ }^{15}$, y las autoridades tradicionales del pueblo Nasa en el departamento del Cauca han llamado la atención por el aumento en el consumo de sustancias, particularmente entre niños y jóvenes ${ }^{16}$. Esto es consecuente con la literatura pertinente a conducta suicida en pueblos indígenas, que ha señalado que la tasa de suicidios en dicha población es superior a la que se observa en la población general, y ha identificado como principal problema asociado al suicidio, el uso de sustancias ${ }^{17}$.

Ahora bien, la adicción a sustancias psicoactivas generalmente trae consigo consecuencias perjudiciales. A nivel social fomenta la destrucción de la familia, la violencia intrafamiliar y el aumento de la criminalidad, lo que a su turno incrementa la morbilidad, la discapacidad y la mortalidad ${ }^{18}$. Igualmente favorece el desarrollo de problemas de salud que van desde la cirrosis, el cáncer y el síndrome de alcoholismo fetal, hasta la depresión y el suicidio ${ }^{19}$. Además, las adicciones tienden a ser causa o efecto de otras

\footnotetext{
${ }^{10}$ Universidad de Antioquia y Organización Indígena de Antioquia, 2006, pp. 137.

${ }^{11}$ Fundación Saldarkiaga Concha, 2019, p. 90.

12 DONDERS, 2015, p. 188.

13 ONIC, 2016.

${ }^{14}$ Instituto Nacional de Medicina Legal y Ciencias Forenses, 2014, p. 16.

15 Departamento de Vaupés, 2015, p. 23.

16 CRIC, 2013.

17 VARgas-Espíndola et al., 2017, p. 131.

18 Carter y Hall, 2013, p. 2.

19 Organización Panamericana de la Salud, 2011, p. 1.
} 
enfermedades mentales: la comorbilidad entre las adicciones y otros trastornos como la depresión, la ansiedad y la psicosis no es inusual (el adicto consume sustancias como mecanismo para aliviar los síntomas asociados a esos otros trastornos ${ }^{20}$ ); asimismo, el uso crónico de sustancias puede propiciar el desarrollo de nuevas enfermedades psiquiátricas ${ }^{21}$.

La literatura concerniente a adicciones nos demuestra así que el incremento en el consumo de alcohol y sustancias psicoactivas representa una amenaza latente para el derecho a la salud y, particularmente, a la salud mental de los pueblos indígenas de Colombia. A continuación examinaremos entonces las normas que resultan relevantes en este contexto, para luego analizar hasta qué punto ellas responden adecuadamente a la situación de adicciones en los indígenas de Colombia.

\section{LA RESPUESTA DEL ORDEN JURÍdiCO: ¿ENTRE LA ABUNDANCIA NORMATIVA Y LA APLICACIÓN DEFICIENTE?}

\section{Del probibicionismo a la adicción como asunto de salud mental}

Desde que las organizaciones internacionales empezaron a formular la política antidrogas, ella se estructuró bajo un enfoque que pretendía disuadir el consumo mediante la penalización ${ }^{22}$. En consonancia con lo anterior, Colombia penalizó no solamente el tráfico, sino también el consumo de sustancias psicoactivas, y de allí que instrumentos como la Ley 30 de 1986 buscaran desincentivar el consumo mediante sanciones penales al consumidor ${ }^{23}$, que incluían el internamiento en establecimientos psiquiátricos por el tiempo necesario para la recuperación ${ }^{24}$.

El modelo prohibicionista empezó a cambiar en Colombia gracias a la Corte Constitucional, que le dio a la drogadicción el calificativo de enfermedad psiquiátrica que afecta la autodeterminación del adicto ${ }^{25}$, y señaló que las adicciones están estrechamente relacionadas con la salud mental ${ }^{26}$ y que es responsabilidad del Estado prestar a los farmacodependientes - a quienes califica como sujetos de especial protección - tratamientos de rehabilitación ${ }^{27}$. De acuerdo con esta jurisprudencia, el Acto legislativo 02 de 2009 estableció que el Estado prestaría especial atención al "enfermo dependiente o adicto” y propendería por su recuperación ${ }^{28}$. Asimismo, la Ley No 1566 de 2012, acerca

\footnotetext{
${ }^{20}$ Carter y Hall, 2013, p. 2.

${ }^{21}$ Carter y Hall, 2013, p. 2.

22 Iregui-Parra et al., p. 6.

${ }^{23}$ Iregui-PARra et al., p. 16.

${ }^{24}$ Ley 30, 1986.

${ }^{25}$ Corte Constitucional, 22.08.2002, T-684/02 y Corte Constitucional, 9.2.2012, T-057/12.

${ }^{26}$ Corte Constitucional, 4.2.2015, T-043/15.

${ }^{27}$ Corte Constitucional, 3.7.2009, T-438/09, Corte Constitucional, 22.5.2015, T-318/15 y Corte Constitucional, 16.9.2016, T-511/16.

${ }^{28}$ Acto legislativo 02, 2009.
} 
de atención integral a consumidores de sustancias psicoactivas, estableció que "el abuso y la adicción deberán ser tratados como una enfermedad que requiere atención integral por parte del Estado" 29 , y en el Plan Nacional para la Promoción de la Salud, Prevención y Atención del Consumo de Sustancias Psicoactivas 2014-2021, el Ministerio de Salud sostuvo que el problema de las drogas debía abordarse como un asunto de salud pública y no como si fuera un delito ${ }^{30}$.

\section{El derecho fundamental a la salud mental}

Debido al estrecho vínculo que existe entre adicciones y salud mental, es posible preguntarse cómo ha abordado el ordenamiento colombiano lo concerniente a la última. La Corte Constitucional señaló en jurisprudencia reciente que para proteger el derecho fundamental a la salud es necesario garantizar la salud tanto física como mental ${ }^{31}$, y por esta razón ha elevado la salud mental a la categoría de derecho fundamental y ha establecido que las personas que padecen trastornos mentales son sujetos de especial protección constitucional $^{32}$. El legislador también ha calificado la salud mental como un derecho fundamental y como tema prioritario de salud pública ${ }^{33}$.

La Corte ha señalado incluso que la enfermedad mental puede constituir discapacidad $^{34}$. A nivel internacional se ha entendido lo mismo y de allí que ante la Comisión Interamericana de Derechos Humanos se hayan solicitado medidas cautelares para proteger la vida e integridad de pacientes psiquiátricos invocando la Convención Interamericana para la Eliminación de todas las Formas de Discriminación contra las Personas con Discapacidad $^{35}$. Fuera del Sistema Interamericano también se ha establecido un vínculo entre enfermedad mental y discapacidad, y por esta razón se ha utilizado la CDPD como instrumento de protección para las personas que padecen trastornos mentales; algunos han argumentado incluso que la CDPD es el instrumento internacional más poderoso para proteger a las personas con dichos trastornos de abusos y discriminación ${ }^{36}$.

\section{El derecho a la salud mental de los indígenas}

Ahora bien, debido a la importancia del derecho fundamental a la salud mental, es preciso indagar si hay normas que protejan ese derecho específicamente para los indígenas. A nivel internacional, el Pacto Internacional de Derechos Económicos, Sociales y Culturales (PIDESC) reconoce en su artículo 12 el derecho al más alto nivel posible

\footnotetext{
${ }^{29}$ Ley $\mathrm{N}^{\circ} 1566,2012$.

30 Ministerio de Salud, 2017, p. 15.

${ }^{31}$ Corte Constitucional, 9.2.2012, T-057/12.

32 Corte Constitucional, 3.7.2015, T-418/15.

${ }^{33}$ Ley N ${ }^{\circ} 1438,2011$ y Ley N ${ }^{\circ} 1616,2013$.

${ }^{34}$ Corte Constitucional, 7.7.2014, T-454/14.

${ }^{35}$ Hillman, 2005, p. 26.

${ }^{36}$ Callard et al., 2012 , p. 193.
} 
de salud física y mental. El CDESC ha afirmado que ese derecho a la salud tiene una dimensión cultural ${ }^{37}$ y ha reclamado una mayor participación de los indígenas en la toma de decisiones en materia de salud ${ }^{38}$. Por su parte, la Convención sobre la Eliminación de todas las Formas de Discriminación Racial (CERD) consagra en su artículo 5 la obligación de los Estados de garantizar igualdad ante la ley para todas las personas en el goce del derecho a la salud ${ }^{39}$.

A su turno, el Convenio 169 de la OIT establece en su artículo 25 que los gobiernos deben velar porque se pongan a disposición de los pueblos indígenas servicios de salud adecuados, o proporcionarles "los medios que les permitan organizar y prestar esos servicios bajo su propia responsabilidad y control para que puedan gozar del más alto nivel de salud física y mental" ${ }^{40}$. Asimismo, la Declaración de las Naciones Unidas sobre los Derechos de los Pueblos Indígenas señala en su artículo 24 que los indígenas "tienen derecho a disfrutar por igual del más alto nivel de salud física y mental" 41 y en su artículo 23 que tienen derecho a participar en la elaboración de los programas de salud y, en lo posible, a administrarlos. Particularmente, en materia de salud mental, los Principios para la Protección de los Enfermos Mentales y el Mejoramiento de la Atención de la Salud Mental de la ONU señalan que "todo paciente tendrá derecho a un tratamiento adecuado a sus antecedentes culturales" $42,43$.

El ordenamiento colombiano ha incorporado varias normas encaminadas a cumplir con sus compromisos internacionales en esta materia. El Decreto 1811 de 1990 estableció que todo programa de salud dirigido a comunidades indígenas debía concertarse previamente con ellas. La Ley 691 de 2001 reglamentó la participación de los grupos étnicos en el Sistema General de Seguridad Social, entre otros, con el fin de garantizar su derecho a la salud. La Ley No 1438 de 2011, que reformó el Sistema General de Seguridad Social en Salud (SGSSS), incorporó como principio de ese sistema el enfoque diferencial, con el fin de reconocer que hay poblaciones que por sus características particulares -incluyendo etnia y raza- requieren garantías especiales para eliminar la discriminación.

En 2014, con el Decreto 1953, el Ministerio del Interior abrió paso al Sistema Indígena de Salud Propio Intercultural (SISPI), que se articularía con el SGSSS para "maximizar los logros en salud de los pueblos indígenas". Según lo anterior, la Ley

37 DONDERS, 2015, p. 181.

38 Mazel, 2016 , p. 347.

${ }^{39}$ Convención sobre la Eliminación de todas las Formas de Discriminación Racial, 1965.

${ }^{40}$ Convenio No 169 sobre Pueblos Indígenas y Tribales, 1989.

${ }^{41}$ Resolución 61/295, 2007.

42 Resolución 46/119, 1991.

${ }^{43}$ El PIDESC, la CERD y el Convenio 169 hacen parte en Colombia del bloque de constitucionalidad, esto es, de aquellas normas que sin hacer parte del texto constitucional, tienen la misma fuerza y jerarquía de la Constitución. La Declaración sobre los Derechos de los Pueblos Indígenas, si bien no tiene la misma fuerza normativa de un tratado internacional, ha sido reconocida por la Corte Constitucional como fuente de derecho, entre otras, en sentencia T-011/19. Y los Principios para la Protección de los Enfermos Mentales y el Mejoramiento de la Atención de la Salud Mental han sido utilizados por esa misma corporación como criterios interpretativos. 
Estatutaria en Salud (1751 de 2015) incluye como elementos esenciales del derecho a la salud la interculturalidad y la protección a los pueblos indígenas, y posteriormente, en 2016, el Ministerio de Salud expediría las “Orientaciones técnicas con enfoque intercultural para la promoción de la salud mental, la prevención del consumo de sustancias psicoactivas y la conducta suicida en población indígena”, un documento de política pública que pretende servir de insumo para reglamentar el SISPI ${ }^{44}$.

\section{La brecha entre el ordenamiento jurídico y su aplicación práctica}

Sin embargo, pese a la gran cantidad de normas que protegen el derecho a la salud mental de toda la población en general, y de los pueblos indígenas en particular, la salud de estos últimos -tanto física como mental- sigue siendo más deficiente que la del resto de la población colombiana. La Corte Constitucional advirtió desde 2009 que, debido al conflicto armado, numerosos pueblos indígenas estaban en peligro de exterminio cultural o físico, y que el desplazamiento causado por el conflicto los ha forzado a vivir en condiciones deplorables, lo que a su turno ha dado paso a la violación de numerosos derechos, entre ellos, el derecho a la salud ${ }^{45}$. Anotó dicha corporación que ante esta crítica situación, las autoridades han respondido mediante la expedición de normas y políticas públicas que pese a tener valor, han tenido repercusiones prácticas precarias ${ }^{46}$.

Desde entonces la situación no ha presentado mejoras significativas: en 2015, en fallo de tutela de segunda instancia, el Tribunal Superior de Arauca ordenó la protección de los derechos fundamentales de distintas comunidades indígenas víctimas de desplazamiento en ese departamento ${ }^{47}$. En su decisión, el Tribunal ordenó a las Entidades Prestadoras de Salud que prestaran a las comunidades Sikuani y Makaguan un servicio de salud con enfoque diferencial ${ }^{48}$. Asimismo, ante la grave situación de alcoholismo y drogadicción que padecen esas comunidades, les ordenó que ejecutaran un proceso de desintoxicación gradual encaminado a su total recuperación ${ }^{49}$.

En 2016 la Contraloría de la República hizo seguimiento al cumplimiento del fallo, pero en su informe notó con preocupación que la situación de los indígenas adictos en situación de calle se había hecho aun peor y concluyó que las entidades accionadas no cumplieron las órdenes del Tribunal, pues todo lo que evidenció fue un "asistencialismo eventual, aislado, sin enfoque diferencial, sin política pública sostenible, carente de recursos económicos y humanos que no logra sacar avante a estas comunidades de la problemática en la que están sumidas ${ }^{50}$.

\footnotetext{
${ }^{44}$ Ministerio de Salud, 2016, p. 7.

45 Corte Constitucional, 26.1.2009, Auto 004 de 2009.

${ }^{46}$ Corte Constitucional, 26.1.2009, Auto 004 de 2009.

47 Tribunal Superior del Distrito Judicial de Arauca, 1.7.2015, Rad. 2014-00052-03.

48 Tribunal Superior del Distrito Judicial de Arauca, 1.7.2015, Rad. 2014-00052-03.

49 Tribunal Superior del Distrito Judicial de Arauca, 1.7.2015, Rad. 2014-00052-03.

${ }^{50}$ Contraloría General de la República, 6.2016.
} 
Ante a la alarmante situación de adicciones en pueblos indígenas de Colombia, nos encontramos entonces frente a una profusa normatividad que, no obstante, tiene pocas repercusiones prácticas, pues en la realidad -como ha sucedido en Arauca- las acciones adoptadas para afrontar esta problemática, o son escasas, o no pasan del simple "asistencialismo eventual" sin vocación de permanencia. Esta situación se explica por múltiples factores que merecen ser investigados a profundidad y que exceden el objeto del presente trabajo. En este artículo nos limitaremos a discutir uno de estos factores, que -según lo argumentaremos a continuación- está asociado a los modelos que se han construido en torno al concepto de adicción, y señalaremos cuál es el entendimiento que se le debería dar a ese concepto para empezar a estructurar soluciones efectivas frente a la problemática de alcoholismo y farmacodependencia en los pueblos indígenas de Colombia.

\section{El MODElo MORAL DE ADICCIÓN VS. LA ADICCIÓN ENTENDIDA COMO ENFERMEDAD: PLANTEAMIENTOS Y LIMITACIONES}

\section{Principales postulados y críticas a cada modelo}

El concepto de adicción ha sido ampliamente debatido en el ámbito ético. Estos debates han influido en el mundo jurídico y de allí que tanto el legislador colombiano como quienes elaboran políticas públicas hayan adoptado distintas posturas para abordar esta problemática. En la primera mitad del siglo XX, la postura ética imperante era la del modelo moral, que entiende el consumo de drogas como una elección -incluso para quienes padecen una adicción-y adopta una posición de reproche frente a esa decisión 51 . Bajo este modelo, el adicto es considerado como una persona egoísta que está dispuesta a perseguir el placer a toda costa; de allí que a la luz de esta postura, el adicto sea culpado por su decisión de consumir drogas y profundamente estigmatizado ${ }^{52}$.

Partiendo de esta aproximación, no resulta sorpresivo que, por lo menos inicialmente, las normas y políticas públicas estuviesen imbuidas de una notoria desaprobación moral hacia el consumo de drogas ${ }^{53}$. Sin embargo, las investigaciones demostraron que aun cuando el placer que experimenta quien consume sustancias psicoactivas tiende a disminuir con el correr del tiempo, ello no disuade al adicto de seguir consumiendo ${ }^{54}$. Esto permitió evidenciar que podría haber otras razones, distintas al egoísmo y al hedonismo, que explicarían la naturaleza de las adicciones. Fue así como surgió el modelo de adicción como enfermedad.

Bajo el nuevo modelo se empezó a entender la adicción como una enfermedad neurobiológica crónica caracterizada por el consumo compulsivo de sustancias psicoactivas,

\footnotetext{
51 PiCKARD, 2017, p. 170.

52 PiCKARD, 2017, p. 170.

53 Carter y Hall, 2013, pp. 1-2.

${ }^{54}$ Kennet et al., 2013, p. 140.
} 
pese a las consecuencias negativas que dicho consumo puede ocasionar ${ }^{55}$. Según sus planteamientos, el uso recurrente de esas sustancias modifica el cerebro a tal punto que para el adicto el consumo se vuelve irresistible, y de allí que no se le pueda culpar por consumir, pues ello no es el producto de una elección sino de una enfermedad ${ }^{56}$. Esta postura ha sido acogida, entre otras, por la Organización Mundial de la Salud, la Asociación Médica Británica y la Asociación Médica Americana ${ }^{57}$. A tal punto ha llegado la aceptación de este modelo que el trastorno por consumo de sustancias está incluido en el Manual Diagnóstico y Estadístico de los Trastornos Mentales (DSM-5) de la Asociación Americana de Psiquiatría ${ }^{58}$.

Así como quedó en evidencia en el acápite anterior, el ordenamiento jurídico colombiano ha transitado del modelo moral al modelo de adicción como enfermedad, y por tanto le ha reconocido al adicto el derecho a recibir tratamiento con miras a su rehabilitación. Esto sin duda constituye un importante avance respecto de la manera en que se abordaba la problemática de las adicciones cuando aún imperaban las normas inspiradas en el modelo moral, pues el nuevo modelo permitió dejar de lado el enfoque punitivo para dar paso a nuevas normas encaminadas a la protección de los derechos fundamentales del adicto y particularmente de su derecho a la salud.

El modelo de adicción como enfermedad, no obstante, ha sido blanco de críticas. Algunos autores sostienen que dicho modelo es contraproducente para el adicto, por cuanto no le permite reconocerse como agente de cambio en su propia recuperación, sino que le lleva a percibirse a sí mismo como dependiente de los profesionales de la salud ${ }^{59}$. Por otra parte, aun cuando se ha demostrado que el abuso de sustancias produce cambios neuroplásticos que dan paso a síntomas de abstinencia, dolor y angustia ${ }^{60}$, hay evidencia de adictos y consumidores habituales que, al alcanzar cierta edad en la que sus responsabilidades laborales o parentales incrementan, dejan de consumir espontáneamente y sin necesidad de tratamiento ${ }^{61}$. La evidencia parece así demostrar que los adictos no están sometidos a un deseo irresistible ${ }^{62}$, lo que llevaría a intuir que hay otras razones que explican la adicción.

Para explorar cuáles podrían ser esas razones, concedamos al modelo moral -en gracia de discusión - que el adicto consume, no por un deseo irresistible, sino por una decisión. Surgen entonces las siguientes preguntas que a continuación intentaremos responder: asumiendo que el adicto consume por una decisión suya ¿es esta una decisión autónoma que puede, por consiguiente, ser objeto de reproche moral? ¿Tiene el adicto

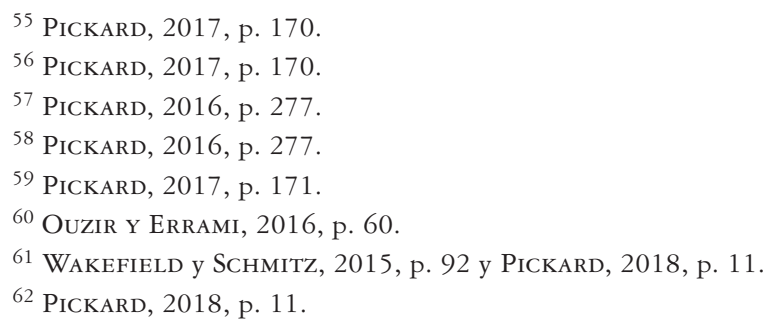


la autonomía para dejar de consumir? Para dar respuesta a estos interrogantes debemos explorar primero el concepto de "autonomía".

\section{El concepto de autonomía}

La autonomía puede entenderse desde la perspectiva del modelo liberal o desde el modelo de autonomía relacional. La autonomía del modelo liberal es eminentemente formal o procedimental, pues considera que el procedimiento mediante el cual una persona adopta una decisión es sumamente importante ${ }^{63}$. Por ello, una decisión autónoma bajo este modelo es aquella donde el sujeto que toma la decisión es capaz de apreciar y entender las opciones disponibles y de manipularlas racionalmente sin interferencia alguna.

Por el contrario, el modelo de autonomía relacional reconoce que todos estamos expuestos a distintas fuerzas sociales en la toma de nuestras decisiones, y por ello autónomo no es quien toma decisiones racionales sin injerencia alguna, sino quien es capaz de responder a esas fuerzas de manera efectiva, sin convertirse en un receptáculo pasivo de las mismas ${ }^{64}$. Una persona vulnerable, a su turno, sería aquella que no está en capacidad de contrarrestar las fuerzas sociales a las que se ve enfrentada y que, por tanto, no puede evitar los acontecimientos que afectan negativamente lo que considera importante ${ }^{65}$. Siguiendo esa lógica, bajo el modelo de autonomía relacional, la vulnerabilidad sería una circunstancia o condición que afecta la capacidad de responder de manera efectiva a las fuerzas sociales y que, consiguientemente, puede socavar la autonomía.

\section{La autonomía en la adicción: ¿modelo liberal o relacional?}

Siguiendo los planteamientos teóricos que dan cuenta del concepto de autonomía, podríamos afirmar que si el modelo moral califica el consumo de sustancias como moralmente reprochable -incluso cuando el consumidor padece una adicción-, es porque considera la adicción como el producto de una decisión autónoma: la decisión de consumir habitualmente. Y si considera que tal decisión es autónoma, es porque la examina desde el modelo liberal de autonomía. En efecto, no es usual que las personas que desarrollan una adicción lo hagan porque un tercero las ha coaccionado para consumir, o porque carecían de capacidad de raciocinio al tomar la decisión de consumir ${ }^{66}$, y de allí que, desde la perspectiva liberal, la adicción pueda verse como el producto de una decisión autónoma prácticamente en todos los casos.

Sin embargo, la decisión del adicto de consumir sustancias psicoactivas tiende a estar más asociada a factores contextuales (abuso, violencia, pobreza, etc.) que a una decisión racional sin injerencia alguna; en efecto, pertenecer a un estatus socioeconómico bajo,

\footnotetext{
63 Christman, 2015.

${ }^{64}$ BARCLAY, 2000, p. 55.

65 Anderson, 2014, p. 3.

${ }^{66}$ Solo en eventos como estos consideraría el modelo liberal que la decisión de consumir no es autónoma.
} 
la falta de integración familiar o haber estado expuesto a patrones de crianza deficiente, entre otros, son factores de riesgo que determinan que una persona sea más proclive a desarrollar adicciones ${ }^{67}$. De allí que el modelo de autonomía relacional-que evalúa la influencia de las fuerzas sociales en la toma de decisiones- parezca más adecuado que el modelo liberal para dar respuesta a las preguntas planteadas en el numeral $1^{\circ}$ del presente acápite. En los párrafos subsiguientes procuraremos responder a esas preguntas desde la óptica de la autonomía relacional, y para ello profundizaremos en el concepto de "vulnerabilidad".

\section{a) Autonomía relacional y vulnerabilidad}

Al presentar el concepto de autonomía relacional, se planteó que la vulnerabilidad es una circunstancia que puede socavar la autonomía. Partiendo entonces de los distintos tipos de vulnerabilidad de que habla la profesora Jackie Scully, indagaremos cómo las vulnerabilidades pueden afectar la autonomía y, por consiguiente, hacer que ciertas personas sean más propensas a desarrollar adicciones.

Para Scully las vulnerabilidades pueden ser inherentes (cuando resultan de una deficiencia física o mental), contingentes (que son el resultado de las barreras sociales que deben enfrentar ciertos grupos, por ejemplo, producto de la discriminación) y ontológicas (que son las que enfrentamos todos como parte inevitable de la vida) ${ }^{68}$.

Para entender mejor en qué consisten las vulnerabilidades ontológicas es preciso citar al profesor Joel Anderson, quien afirma que por vivir en sociedad, los seres humanos necesitamos ciertas competencias sociales para ser realmente autónomos; competencias que se desarrollan mediante relaciones intersubjetivas de reconocimiento recíproco ${ }^{69}$. Sin embargo, debido a que nada garantiza que obtendremos ese reconocimiento -pues al entablar relaciones con otras personas podemos encontrarnos con actitudes denigrantes o irrespetuosas- ${ }^{70}$, todos somos ontológicamente vulnerables a la ausencia de reconocimiento. No significa esto que dicha vulnerabilidad ontológica socave siempre la autonomía; no obstante, si el riesgo de ausencia de reconocimiento se materializa, puede llegar a socavarla.

Veamos a continuación cómo pueden interactuar los distintos tipos de vulnerabilidad para hacer que determinados grupos sociales -como los indígenas en Colombia- sean especialmente vulnerables a desarrollar adicciones, y para ello tomemos como ejemplo el caso de los Sikuani en Arauca.

${ }^{67}$ TeEson et al., 2012, p. 46.

68 También habla de vulnerabilidades globales atribuidas que no es del caso discutir en el presente artículo (SCULLY, 2013, pp. 206-209).

${ }^{69}$ Anderson, 2014, pp. 5, 8.

70 Anderson, 2014, pp. 9-10. 


\section{b) Vulnerabilidades y adicción: el caso de los Sikuani}

Antes de la conquista española, los Sikuani -un grupo indígena de la familia Guahibo- eran nómadas y vivían de la caza, la recolección ${ }^{71}$ y el intercambio de bienes. Una vez que Colombia alcanzó la independencia, se inició un proceso espontáneo de colonización de los llanos orientales ${ }^{72}$. La invasión por parte de los llamados “colonos" afectó drásticamente la vida de los indígenas, no solo por los conflictos por el control territorial, sino por la transformación del medio ambiente y la dramática reducción de las especies salvajes que trajo consigo la ganadería extensiva introducida por ellos ${ }^{73}$. Esto impactó negativamente la dieta de los indígenas, quienes en un intento de preservar su hábitat y encontrar nuevas fuentes de alimentación, empezaron a cazar el ganado, bien fuera para consumirlo o para evitar que siguiera alterando el equilibrio ecosistémico ${ }^{74}$. En retaliación, los colonos y terratenientes iniciaron la caza sistemática de los indígenas, mediante una práctica conocida como "guahibiadas" -que pronto alcanzaría aceptación entre la "sociedad civilizada"75 -, por considerar que los intentos de los Guahibos por detener los avances de la colonización eran un reflejo de su comportamiento 'inmoral' y "salvaje" 76 .

Para evitar los conflictos entre colonos e indígenas, el gobierno creó resguardos, cuya área se redujo con las constantes migraciones desde el centro hacia el oriente del país, y de allí que para la década de los setenta fuese posible encontrar una sola propiedad de hasta 60.000 hectáreas, mientras que el área total de los resguardos indígenas únicamente alcanzaba las 14.000 hectáreas ${ }^{77}$. Fue así como el pueblo Sikuani, que alguna vez fue nómada, se volvió sedentario a la fuerza.

La historia de violencia y abuso no termina allí. Con el descubrimiento del pozo petrolero de Caño Limón en Arauca en 1983, la región se volvió económicamente atractiva y se convirtió en escenario de nuevos conflictos entre grupos guerrilleros, paramilitares y el Estado ${ }^{78}$, que llevaron a que el área de los resguardos se redujera aún más, y que expusieron a los indígenas al consumo de alcohol y sustancias psicoactivas. Hoy -como lo referimos en el tercer capítulo- el alcoholismo y la drogadicción representan un serio problema de salud pública para los Sikuani.

No es difícil observar cómo esta historia de colonización exacerbó las vulnerabilidades de los Sikuani y consiguientemente socavó su autonomía, creando el caldo de cultivo perfecto para la problemática de adicciones. Efectivamente, desde sus primeras interacciones con los españoles y posteriormente con los colonos, los Sikuani recibieron

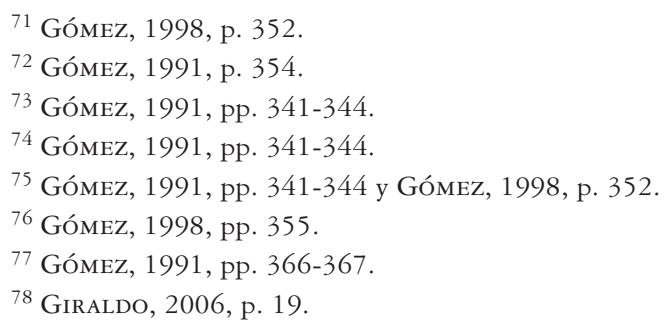


un trato inhumano. De allí que las relaciones que entablaron con sus invasores no fuesen de reconocimiento recíproco; para los Sikuani la vulnerabilidad ontológica se materializó en una ausencia de reconocimiento que con el tiempo se volvió la regla. Los Sikuani se convirtieron en un grupo históricamente marginado que aún hoy experimenta vulnerabilidades contingentes asociadas a las barreras socioeconómicas que resultan de la discriminación.

Ahora bien, como lo referimos en el primer capítulo, es común que ciertas minorías étnicas, como los pueblos indígenas -y en este caso los Sikuani- estén en los niveles socioeconómicos más bajos, y ello ha llevado a que tengan una salud mental deficiente ${ }^{79}$. El contexto socioeconómico es en sí mismo un factor de riesgo que puede contribuir al desarrollo de adicciones ${ }^{80}$; sin embargo, la salud mental deficiente (potencialmente causada por las vulnerabilidades contingentes) es también un factor de riesgo, pues se ha demostrado que las enfermedades psiquiátricas hacen que el cerebro sea más susceptible a la adicción ${ }^{81}$ y, consiguientemente, quienes padecen dichas enfermedades son inherentemente más vulnerables a desarrollar adicciones.

El caso de los Sikuani es el de muchos otros pueblos indígenas de Colombia en los que las vulnerabilidades ontológicas, contingentes e inherentes confluyeron para socavar su autonomía y hacerlos más vulnerables a caer en la adicción. Este caso nos permite concluir que, aunque desde el modelo liberal de autonomía la adicción sea vista como el producto de una decisión autónoma que puede ser objeto de reproche moral, bajo la óptica de la autonomía relacional -más apropiada para este contexto- el adicto no llega a la adicción por una decisión plenamente autónoma, pues su decisión de consumir sustancias suele tener su origen en distintos tipos de vulnerabilidades que concurren para erosionar - mas no extinguir- su autonomía. Con esto damos respuesta a la primera pregunta planteada en el numeral $1^{\circ}$ del presente acápite.

¿Y qué decir de la segunda? ¿Tiene el adicto la autonomía para dejar de consumir? Según la propuesta de la profesora Hana Pickard, aquellos para quienes las adicciones se convierten en un problema crónico, normalmente son personas de contextos socioeconómicos deprimidos (vulnerabilidad contingente) o que padecen otros trastornos mentales (vulnerabilidad inherente), para quienes el alcohol y las drogas ofrecen una alternativa menos onerosa para afrontar la aflicción y la miseria ${ }^{82}$. Para ellos estas sustancias cumplen una función psicológica ${ }^{83}$. Si esto fuese así, únicamente si las vulnerabilidades que explican el origen de la adicción son superadas, podría el adicto alcanzar la autonomía necesaria para dejar de consumir. Es decir, que solo sería plenamente autónomo para salir de la adicción cuando tenga otras alternativas disponibles - distintas a la "automedicación"- para aliviar su sufrimiento.

Siguiendo el razonamiento así expuesto, parece ser que tanto el modelo moral como el modelo de adicción como enfermedad se quedan cortos: pues el consumo del adicto no

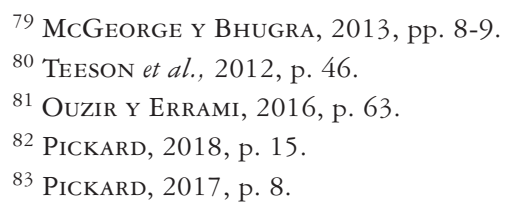


estaría motivado exclusivamente por un deseo compulsivo irresistible-como lo sugiere el modelo de adicción como enfermedad-, pero tampoco por una decisión plenamente autónoma y moralmente reprochable -como lo pretende el modelo moral-.

\section{V. ¿CÓMO ENTENDER LA ADICCIÓN? El Planteamiento Ético de Hana Pickard}

Tomando como base la línea argumentativa expuesta, ¿cómo abordar entonces el problema de la adicción?, a continuación expondremos la que consideramos es la mejor alternativa conceptual para entender dicho fenómeno y la que - de ser acogida- permitiría avanzar en la consecución de soluciones para la situación de adicciones en pueblos indígenas de Colombia. Para el efecto partiremos del planteamiento ético de la profesora Hana Pickard, quien analiza la adicción desde el marco conceptual que denomina "responsabilidad sin reprobación" 84 . Con ello busca abolir la censura hacia el adicto sin negarle su carácter de agente moral, destaca la importancia de asumir una actitud compasiva hacia quien padece una adicción, y propende por la justicia social, para así evitar que la injusticia se convierta en ambiente propicio para el desarrollo de adicciones.

El modelo de adicción como enfermedad ya había contribuido significativamente en la lucha contra la estigmatización del adicto, pues permitió reconocer que el consumo prolongado de sustancias desencadena un proceso patológico que afecta la capacidad de controlar dicho consumo ${ }^{85}$. Con ello, el uso de sustancias dejó de verse como una decisión autónoma del adicto que podía ser objeto de reproche moral.

Ahora bien, como se planteó en el capítulo anterior, la autonomía del adicto en efecto puede verse reducida por la confluencia de distintas vulnerabilidades y por los cambios neuroplásticos que genera el consumo; sin embargo, reducción no significa extinción ${ }^{86} \mathrm{y}$, por tanto, so pretexto de combatir el estigma, no se le puede negar al adicto su calidad de agente moral alegando que la adicción no es más que el producto de un deseo compulsivo irresistible.

No obstante, aun cuando al adicto no se le pueda negar su carácter de agente moral, si su autonomía se encuentra reducida, la decisión de consumir tampoco puede ser objeto de reproche moral, pues ello sería abiertamente injusto. Hemos dicho que el origen de la adicción se encuentra en gran parte en las circunstancias socioeconómicas y las oportunidades limitadas, y de allí que gran cantidad de adictos vengan de contextos deprimidos, grupos marginados y minorías étnicas ${ }^{87}$. Si ello es así, la adicción deja de ser un problema exclusivo del adicto para convertirse en un problema social frente al cual tanto el Estado como la sociedad deben asumir responsabilidad, por haber tolerado

\footnotetext{
${ }^{84}$ Responsibility without blame.

${ }^{85}$ KennetT, 2013, p. 3.

86 PICKARD, 2017, p. 8.

${ }^{87}$ PICKARD, 2017, p. 10.
} 
la existencia de un contexto socieconómico que se ha convertido en caldo de cultivo de adicciones para ciertos grupos poblacionales, como los indígenas.

Así pues, la aproximación frente a la problemática de adicciones no puede ser ni el reproche del adicto -como lo sugiere el modelo moral-, ni el enfoque exclusivo en corregir una deficiencia intrínseca suya -como lo sugiere el modelo de adicción como enfermedad-, sino que las normas y políticas públicas respecto de esta materia deben apuntar a remediar las carencias y desigualdades socioeconómicas que contribuyen al surgimiento de la adicción, además de brindarle tratamiento al adicto sin negarle su carácter de agente moral capaz de gestar cambios en su propia vida.

Visto así, los dos modelos que hasta el momento han inspirado las normas jurídicas parecen ser una cómoda salida que nos permite cerrar los ojos frente a la responsabilidad que nos corresponde como sociedad frente al fenómeno de las adicciones ${ }^{88}$, y de allí que sea este el momento de entender dicha problemática en su real dimensión y de reconocer la deuda que tenemos frente a ciertos grupos, como los pueblos indígenas. Un Estado capaz de entender la adicción de esta manera, tal vez pueda pasar del "asistencialismo eventual" y la normatividad sin repercusiones prácticas, a un escenario de acciones concretas con efectos palpables. Un Estado capaz de reconocerse como responsable -al menos parcialmente- de este fenómeno, tal vez pueda entender que brindarle asistencia a los indígenas adictos y adoptar medidas para mejorar su contexto socioeconómico no es un asunto de caridad sino de justicia social.

En el siguiente capítulo exploraremos las razones por las que consideramos que, de las normas referidas en el segundo capítulo, la CDPD es la que más se acopla a la manera de entender la adicción expuesta en el presente artículo y, por esta razón, la que ofrece el marco normativo más adecuado para comprender la problemática de adicciones en los pueblos indígenas de Colombia.

\section{LAS ADICCIONES EN PUEBLOS INDÍGENAS VISTAS DESDE LA ÓPTICA DE LA CDPD}

La CDPD señala en su artículo $1^{\circ}$ que "las personas con discapacidad incluyen a aquellas que tengan deficiencias físicas, mentales, intelectuales o sensoriales a largo plazo que, al interactuar con diversas barreras, puedan impedir su participación plena y

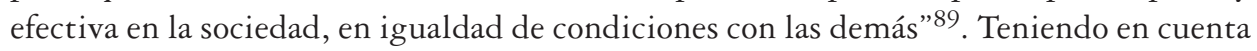
la definición de discapacidad implícita en este artículo, ¿puede la adicción subsumirse en esa definición?

En lo que a la deficiencia concierne, es cierto que el consumo prolongado de sustancias psicoactivas provoca cambios neuroplásticos que afectan la capacidad de controlar el uso de dichas sustancias. Esto ciertamente podría calificarse como una deficiencia. Por

\footnotetext{
88 PiCKARD, 2017, p. 10.

${ }^{89}$ Convención sobre los Derechos de las Personas con Discapacidad, 2006.
} 
su parte, en lo que atañe a las "barreras sociales" experimentadas por el adicto, también es cierto que los adictos enfrentan estigma y estereotipos que impactan sus vidas negativamente y limitan su participación en la sociedad y sus oportunidades de empleo ${ }^{90}$. Vemos pues cómo, de acuerdo con lo señalado en el artículo $1^{\circ}$, la deficiencia y las barreras sociales pueden interactuar para "impedir [la] participación plena y efectiva [del adicto] en la sociedad, en igualdad de condiciones con las demás”. Parecería entonces que la adicción puede subsumirse en la categoría de discapacidad y, consiguientemente, puede analizarse desde la óptica de la CDPD.

La legislación contemporánea respecto de discapacidad de hecho suele incluir la adicción en esa categoría, buscando con ello eliminar el estigma hacia el adicto ${ }^{91}$. Se ha señalado en efecto que examinar la adicción desde la perspectiva de la discapacidad (entendida esta desde el modelo social de discapacidad) permite comprender mejor los factores sociales y estructurales que conducen a ella y, por esta razón, permite adoptar soluciones más holísticas y no puramente biomédicas ${ }^{92}$.

No significa lo anterior que la consecuencia ineludible de entender la adicción como enfermedad sea la inobservancia de los determinantes sociales y económicos de la adicción. Hay enfoques de salud -como la atención primaria93 por un abordaje global de la salud. Sin embargo, la influencia del modelo de adicción como enfermedad en la formulación de leyes y políticas públicas tiende a traer consigo el diseño de soluciones eminentemente biomédicas ${ }^{94}$. Si bien el modelo en cuestión permitió quitarle fuerza al reproche moral que recae en el adicto, no ha sido suficiente para lograr soluciones omnicomprensivas para esta problemática.

El análisis de la adicción desde el lente de la discapacidad cumple entonces un objetivo político; así como subsumir una determinada condición en la categoría de enfermedad cumple un propósito político - pues permite demandar servicios médicos para superar esa condición ${ }^{95}$ - subsumirla en la categoría de discapacidad (vista desde el modelo social de discapacidad) permitiría hacer demandas a distintos sectores $-\mathrm{y}$ no solamente al sector salud- para remover las barreras socioeconómicas que explican el origen de esa condición. Es esto entonces lo que se busca al atribuirle a la adicción el calificativo de discapacidad.

Ahora bien, ¿por qué decimos que la CDPD es el instrumento normativo que más se acopla a la manera de entender la adicción propuesta en este artículo? Si bien la Convención reconoce la existencia de una deficiencia en quienes padecen una discapacidad y por tanto consagra en favor suyo un derecho a la habilitación y rehabilitación,

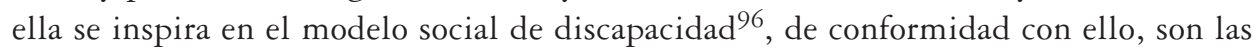

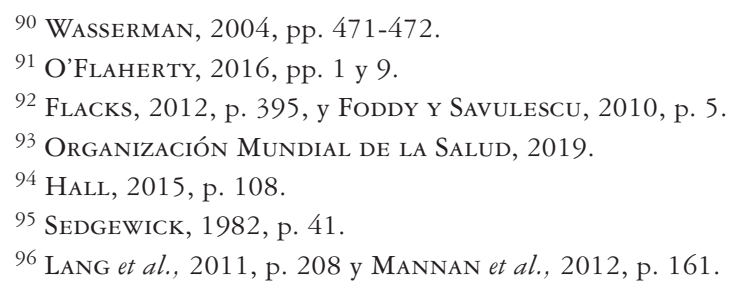


instituciones y estructuras sociales -y no las deficiencias del individuo- las que causan la discapacidad ${ }^{97}$, y por eso busca remover las barreras que impiden la efectiva participación en sociedad de las personas con discapacidad. Este modelo se diferencia del modelo médico, que sí considera la discapacidad como un problema del individuo causado por una condición médica que debe ser objeto de tratamiento ${ }^{98}$. El modelo social de discapacidad, en concordancia con los planteamientos del modelo de autonomía relacional, reconoce que las personas están inmersas en un entramado de fuerzas sociales que pueden fortalecer su autonomía o limitarla y, por tanto, permite identificar el rol que cumple la sociedad y sus actitudes, las políticas públicas y la misma ley en el surgimiento de la discapacidad 99 .

La remoción de barreras por la que propende la CDPD se ha traducido primordialmente en obligaciones de accesibilidad e implementación de ajustes razonables ${ }^{100}$; sin embargo, a causa de la importancia que le atribuye la Convención al papel de la sociedad en la discapacidad, dicho instrumento también ha suscitado importantes reflexiones en torno al rol de las circunstancias socioeconómicas en el origen de condiciones discapacitantes. Si bien este tipo de reflexiones no generan cambios inmediatos, sí permiten dejar de ver la discapacidad como un fenómeno indivual, para en cambio dirigir la mirada hacia factores estructurales que explican su origen. Dichas reflexiones son el primer paso para un cambio de paradigma y un abordaje de la discapacidad que preste especial atención a sus causas estructurales.

Algunas de las reflexiones en cuestión han señalado que la CDPD permite reconocer el papel de la pobreza, la raza y otros factores en el desarrollo de condiciones incapacitantes $^{101}$. En efecto, se ha demostrado que la discriminación racial contribuye a la creación de desventajas socioeconómicas y tiene un impacto negativo en la salud mental y física de las personas ${ }^{102}$. La CDPD permite así dar cuenta de esas circunstancias para abrir paso a soluciones que, en vez de enfocarse exclusivamente en corregir las deficiencias del individuo, propendan por desarrollar políticas sociales y económicas para fortalecer las capacidades de las comunidades, las familias y los individuos ${ }^{103}$.

En el ámbito de la salud mental en el que se circunscribe la problemática de las adicciones se han suscitado reflexiones especialmente interesantes. A este respecto se ha dicho que el modelo social de discapacidad -y la CDPD que en él se inspira-, antes que pensar en el trastorno mental como un fenómeno descontextualizado, busca identificar y dar solución a los procesos de opresión social y discriminación ${ }^{104}$ que llevan al deterioro de la salud mental. Este modelo propende así por una aproximación integral al trastorno

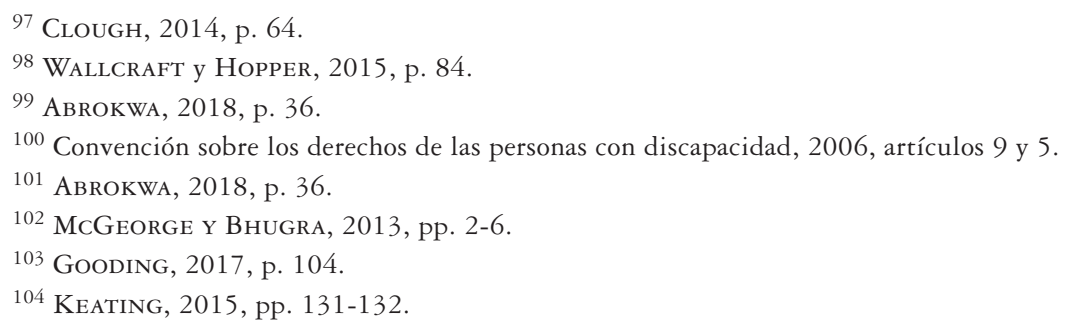


mental, que va más allá del tratamiento psiquiátrico e incorpora soluciones en materia de vivienda, empleo y educación, entre otros ${ }^{105}$.

Respecto del problema concreto que nos ocupa: las adicciones en pueblos indígenas de Colombia, podemos decir que si tal problemática fuese abordada desde la óptica de la CDPD, ello permitiría pensar en soluciones que, en vez de enfocarse exclusivamente en brindarle tratamiento médico al adicto (o en penalizar su decisión de consumir), den prioridad a la remoción de las vulnerabilidades contingentes -o barreras socioeconómicas- que fomentan el desarrollo de adicciones y limitan la autonomía de quienes ya las padecen.

Teniendo en mente lo anterior, consideramos que la CDPD es el instrumento que ofrece el marco normativo más adecuado para comprender en su real dimensión la problemática de adicciones en los pueblos indígenas de Colombia. En efecto, al estar inspirada en el modelo social de discapacidad, la CDPD invita a reflexionar acerca del papel de la sociedad y del Estado en el surgimiento de esta problemática y también a pensar en soluciones no solamente enfocadas en el tratamiento individual del adicto, sino también en fortalecer su autonomía mediante la remoción de las vulnerabilidades contingentes $-\mathrm{O}$ barreras sociales- que explican el origen de la misma. Al reconocer entonces que la sociedad es al menos parcialmente responsable de la proliferación de la adicción, la adopción de las soluciones en cuestión deja de ser un asunto de caridad, para convertirse en una exigencia de justicia social.

\section{CONCLUSIÓN}

En los capítulos precedentes hemos argumentado que una de las principales razones detrás del fenómeno de desconexión entre el panorama normativo y la realidad en relación con la problemática de adicciones en los pueblos indígenas de Colombia, está ligada a las limitaciones de los modelos respecto de la naturaleza de la adicción que han permeado el ordenamiento jurídico colombiano. En respuesta a ello sostuvimos que una recta comprensión de la naturaleza de la adicción exige entender que ella es el resultado de vulnerabilidades socialmente causadas, que confluyen para socavar -mas no extinguir- la autonomía del individuo, y de allí que una adecuada solución a esa problemática exija reconocer nuestro papel como sociedad en el surgimiento de esas vulnerabilidades, para así combatirlas y brindarle tratamiento al adicto sin negarle su carácter de agente moral capaz de transformar su propia vida.

Efectivamente, aunque ya hay políticas públicas que reconocen la importancia de la inclusión social y la equidad para la rehabilitación de las personas con trastornos mentales ${ }^{106}$, es preciso que tanto la sociedad como las autoridades encargadas de aplicar las normas y políticas, vean las limitaciones del modelo moral y del modelo de adicción

105 Keating, 2015, pp. 131-132.

106 Ministerio de SAlud, 2015. 
como enfermedad, pues solo en el momento en que la sociedad y el Estado sean capaces de reconocer su papel en el surgimiento de la adicción, será posible pasar del "asistencialismo eventual" y la normatividad sin impacto real al escenario de las soluciones concretas con repercusiones tangibles. Para esos efectos, la CDPD puede resultar de gran utilidad, y de allí que sea este el momento de empezar a explotar el potencial de dicho instrumento en el análisis de la problemática de adicciones en los pueblos indígenas de Colombia.

\section{BiBLIOGRAFÍA}

Aвrokwa, Alice, 2018: "When They Enter, We All Enter: Opening the Door to Intersectional Discrimination Claims Based on Race and Disability", Michigan Journal of Race E Law, volumen 24, $\mathrm{N}^{\circ}$ 1. Disponible en HTTPS:/REPOSITORY.LAW.UMICH.EDU/MJRL/ VOL24/ISS1/3/. [Fecha de consulta 12.2.2020].

Anderson, Joel, 2014: “Autonomy and vulnerability entwined”, en Wendy Rogers, Catriona Mackenzie y Susan Dodds (editoras), Vulnerability: New essays in ethics and feminist philosophy, Oxford, Oxford University press, pp. 1-35. Disponible en HTTPS://WWW.OXFORDSCHOLARSHIP.COM/VIEW/10.1093/ACPROF:OSO/9780199316649.001.0001/ ACPROF-9780199316649. [Fecha de consulta 18.1.2020].

BARClAy, Linda, 2000: "Autonomy and the social self", en Catriona Mackenzie y Natalie Stoljar (editoras), Relational autonomy: Feminist perspectives on autonomy, agency, and the social self, New York, Oxford University Press, pp. 52-71.

Callard, F., Sartorius, N., Arboleda-Flórez, J., Bartlett, P., Helmchen, H., Stuart, H., Taborda, J., Graham, T., 2012: Mental Illness, Discrimination and the Law, Oxford: Wiley-Blackwell.

Carter, Adrian y Hall, Wayne, 2013: "The Rights of Individuals Treated for Drug, Alcohol, and Tobacco Addiction”, en Michael Dudley, Derrick Silove, and Fran Gale (editores), Mental Health and Human Rights: Vision, praxis, and courage, Oxford, Oxford University Press, pp. 1-16. Disponible en HTTPS://OXFORDINDEX.OUP.COM/VIEW/10.1093/ MED/9780199213962.003.0008. [Fecha de consulta 6.1.2020].

Christman, John (2018). "Autonomy in moral and political philosophy". Recuperado el 18.1.2020, de Stanford Encyclopedia of Phylosophy: HTTPS://PLATO.STANFORD.EDU/ ENTRIES/AUTONOMY-MORAL/.

Clough, Beverley, 2014: “People Like That': Realising The Social Model In Mental Capacity Jurisprudence". Medical Law Review, volumen 23, No 1. Disponible en HTTPS://ACADEMIC.OUP.COM/MEDLAW/ARTICLE-ABSTRACT/23/1/53/1038870. [Fecha de consulta 12.2.2020].

CRIC (7 de octubre de 2013). Pronunciamiento de las autoridades indígenas frente a los cultivos de uso ilícito. Recuperado el 8.1.2020, de CRIC: HTTPS://WWW.CRIC-COLOMBIA.ORG/PORTAL/ PRONUNCIAMIENTO-DE-LAS-AUTORIDADES-INDIGENAS-FRENTE-AL-LOS-CULTIVOS-DE-USO-ILICITO/.

Departamento de Vaupés (2015). Plan departamental de reducción de la oferta de sustancias psicoactivas. Recuperado el 6.1.2020, de Observatorio de drogas de Colombia: HTTP:// WWW.ODC.GOV.CO/PORTALS/1/POLITICA-REGIONAL/DOCS/PLAN-DEPARTAMENTAL-DROGAS-VAUPES.PDF.

Donders, Yvonne, 2015: "Exploring the Cultural Dimensions of the Right to the Highest Attainable Standard of Health", Potchefstroom Electronic Law Journal, volumen 18, No 2. 
Disponible en HTTP://WWW.SCIELO.ORG.ZA/SCIELO.PHP?SCRIPT=SCI_ARTTEX$T \& P I D=S 1727-37812015000200006$. [Fecha de consulta: 8.1.2020].

Flacks, Simon, 2012: "Deviant Disabilities: The Exclusion of Drug and Alcohol Addictions from the Equality Act 2010", Social E Legal Studies, volume 21, No 3. Disponible en HTTPS://JOURNALS.SAGEPUB.COM/DOI/FULL/10.1177/0964663912437991. [Fecha de consulta: 13.6.2020].

French, Laurence, 2004: “Alcohol and Other Drug Addictions Among Native Americans", Alcoholism Treatment Quarterly, volume 22, $\mathrm{N}^{\circ}$ 1. Disponible en HTTPS://WWW.TANDFONLINE.COM/DOI/ABS/10.1300/J020V22N01_06. [Fecha de consulta: 11.6.2020].

Foddy, Bennett y Savulescu, Julian, 2015: "Relating Addiction to Disease, Disability, Autonomy, and the Good Life", Philosophy, Psychiatry, E Psychology, volume 17, $\mathrm{N}^{\circ} 1$. Disponible en HTTPS://MUSE.JHU.EDU/ARTICLE/380813. [Fecha de consulta: 13.6.2020].

Fundación SALdARRIaga Concha (2019). Estigma y discapacidad psicosocial en el marco de los resultados en salud mental del conflicto armado en Colombia. Foco particular población indígena y afrodescendiente. Recuperado el 8.1.2020, de Fundación Saldarriaga Concha: HTTPS://WWW.SALDARRIAGACONCHA.ORG/ INVESTIGACION-ESTIGMA-Y-DISCAPACIDAD-PSICOSOCIAL/.

GIRALDO, Germán, 2006: La colonización en la Orinoquia colombiana: Arauca (1900-1980), Bogotá: Ediciones Antropos.

Gómez, Augusto, 1998: “Cuiviadas y Guajibiadas. La guerra de exterminio contra los grupos indígenas cazadores-recolectores de los Llanos Orientales (Siglos XIX y XX)”, Anuario Colombiano De Historia Social Y De La Cultura, volumen 25. Disponible en HTTP://WWW.BDIGITAL. UNAL.EDU.CO/20555/1/16710-52314-1-PB.PDF. [Fecha de consulta: 18.1.2020].

Gómez, Augusto, 1991: Indios, colonos y conflictos: Una bistoria regional de los Llanos Orientales, 1870-1970, Bogotá: Siglo XXI Editores.

Hall, W., Carter, A., y Forlini, C., 2015: "The brain disease model of addiction: is it supported by the evidence and has it delivered on its promises", Lancet Psychiatry, volume 2, $\mathrm{N}^{\circ}$ 1. Disponible en HTTPS://WWW.THELANCET.COM/PDFS/JOURNALS/LANPSY/ PIIS2215-0366(14)00126-6.PDF. [Fecha de consulta: 12.6.2020].

Hillman, Alison, 2005: "Protecting Mental Disability Rights: A Success Story in the InterAmerican Human Rights System”, Human Rights Brief, volumen 12, No 3. Disponible en HTTPS://DIGITALCOMMONS.WCL.AMERICAN.EDU/HRBRIEF/VOL12/ISS3/7/. [Fecha de consulta: 10.1.2020].

Instituto Nacional de Medicina Legal (2014). Boletín Epidemiológico - Suicidio de indígenas en Colombia - 2010-2014. Recuperado el 6.1.2020, de Instituto Nacional de Medicina Legal y Ciencias Forenses: HTTPS:/WWW.MEDICINALEGAL.GOV.CO/DOCUMENTS/20143/57952/ SUICIDIO+DE+IND\%C3\% ADGENAS+EN+COLOMBIA + 2010-2014.PDF.

Iregui-Parra, P., Palacios-Sanabria, M., Torres-Villarreal, M., Moreno, S., TrianaAnCinez, B., El consumo de drogas en Colombia: una reflexión desde el derecho internacional de los derechos humanos, Bogotá: Universidad de los Andes, Facultad de Economía.

Keating, Frank, 2015: "Linking 'race', mental health and a social model of disability: what are the possibilities?”, en Helen Splandler, Jill Anderson y Bob Sapey (editores), Madness, Distress and the Politics of Disablement, Bristol, The Policy Press, pp. 127-138.

Kennet, Jeanette, 2013: “Addiction, choice and disease”, en Nicole Vincent (editora), Neuroscience and legal responsibility, New York, Oxford University Press, pp. 1-30. Disponible en HTTPS://WWW.OXFORDSCHOLARSHIP.COM/VIEW/10.1093/ACPROF:OSO/9780199925605.001.0001/ACPROF-9780199925605-CHAPTER-11. [Fecha de consulta: $13 \cdot 6.2020]$. 
Kennet, J., Matthews, S., Snoek, A., 2015: "Pleasure and addiction”, en Hanna Pickard, Serge Ahmed y Bennett Foddy (editores), Alternative Models of Addiction, Lausanne, Frontiers in Psychiatry, pp. 131-141. Disponible en: HTTPS://WWW.FRONTIERSIN.ORG/ RESEARCH-TOPICS/1036/ALTERNATIVE-MODELS-OF-ADDICTION. [Fecha de consulta: 13.1.2020].

Lang, R., Kett, M., Groce, N., y Trani, J., 2011: "Implementing the United Nations Convention on the rights of persons with disabilities: Principles, implications, practice and limitations", ALTER, volumen 5. Disponible en: HTTPS://DOI.ORG/10.1016/J. ALTER.2011.02.004. [Fecha de consulta: 13.6.2020].

Mannan, H., Maclachlan, M., McVeigh, J., y The EquitAble Consortium, 2012: "Core concepts of human rights and inclusion of vulnerable groups in the United Nations Convention on the rights of persons with disabilities", ALTER, volumen 6. Disponible en: HTTPS://DOI.ORG/10.1016/J.ALTER.2012.05.005. [Fecha de consulta: 13.6.2020].

Mazel, Odette, 2016: "Self-Determination and the Right to Health: Australian Aboriginal Community Controlled Health Services”, Human Rights Law Review, volumen 16. Disponible en: HTTPS://ACADEMIC.OUP.COM/HRLR/ARTICLE-ABSTRACT/16/2/323/2356218. [Fecha de consulta: 11.1.2020].

McGeorge, Tristan y Bhugra, Dinesh, 2013: "Race Equality in Mental Health", en Michael Dudley, Derrick Silove, and Fran Gale (editores), Mental Health and Human Rights: Vision, praxis, and courage, Oxford, Oxford University Press, pp. 1-19. Disponible en HTTPS:// OXFORDINDEX.OUP.COM/VIEW/10.1093/MED/9780199213962.003.0008. [Fecha de consulta 6.1.2020].

Ministerio de SALUd (2015). Lineamientos nacionales para la implementación de RBC en salud mental. Recuperado el 19.2.2020, de Minsalud: HTTPS:/WWW.MINSALUD.GOV.CO/ SITES/RID/LISTS/BIBLIOTECADIGITAL/RIDE/VS/PP/ENT/LINEAMIENTOS-NACIONALES-RBC.PDF.

Ministerio DE SALUd (2OI6). Orientaciones técnicas con enfoque intercultural para la promoción de la salud mental, la prevención del consumo de sustancias psicoactivas y la conducta suicida en población indígena. Recuperado el 6.1.2020, de Minsalud: HTTPS://WWW. MINSALUD.GOV.CO/SITES/RID/LISTS/BIBLIOTECADIGITAL/RIDE/VS/PP/ENT/ SALUD-MENTAL-INDIGENA-ORIENTACIONES-TECNICAS.PDF.

Ministerio de SALUd (2017). Plan nacional para la promoción de la salud, la prevención, y la atención del consumo de sustancias psicoactivas 2014-2021. Recuperado el 9.1.2020, de Minsalud: HTTPS://WWW.MINSALUD.GOV.CO/SITES/RID/LISTS/BIBLIOTECADIGITAL/ RIDE/VS/PP/PLAN-NACIONAL-CONSUMO-ALCOHOL-2014-2021.PDF.

ONIC (12 de agosto de 2016). ¡La juventud indígena somos semilla de resistencia, unidad y paz! Recuperado el 8.1.2020, de ONIC: HTTPS://WWW.ONIC.ORG.CO/ COMUNICADOS-ONIC/1391-LA-JUVENTUD-INDIGENA-SOMOS-SEMILLA-DE-RESISTENCIA-UNIDAD-Y-PAZ.

Organización Mundial de la SAlud (2019), Atención primaria. Recuperado el 12.6.2020, de Organización Mundial de la Salud: HTTPS://WWW.WHO.INT/ES/NEWS-ROOM/ FACT-SHEETS/DETAIL/PRIMARY-HEALTH-CARE

Organización Panamericana de la Salud (2011). Alcohol y salud de los pueblos indígenas. Recuperado el 6.1.2020, de OPS: HTTPS:/WWW.PAHO.ORG/HQ/DMDOCUMENTS/2009/ ALCOHOLYSALUDINDIGENA06.PDF.

O’Flaherty, Jake, 2016: "Addiction as Disability: Implications within Legislation" The Post, volume 2, No 1 . Disponible en HTTPS://POST.LAKEHEADU.CA/ARTICLE/VIEW/1463. [Fecha de consulta: 13.6.2020]. 
Ouzir, Mounir y Errami, Mohammed, 2016: "Etiological theories of adicction: A comprehensive update on neurobiological, genetic and behavioural vulnerability" Pharmacology Biochemistry and Behavior, volumen 148. Disponible en HTTPS://WWW.SCIENCEDIRECT.COM/ SCIENCE/ARTICLE/PII/S0091305716301046. [Fecha de consulta: 17.1.2020].

PiCKARD, Hanna, 2016: "Denial in Addiction”, Mind E Language, volumen 31, N³. Disponible en HTTPS://ONLINELIBRARY.WILEY.COM/DOI/10.1111/MILA.12106. [Fecha de consulta: 13.1.2020].

Pickard, Hanna, 2017: "Responsibility without Blame for Addiction", Neuroetbics, volumen 10, No 1 . Disponible en HTTPS:/LINK.SPRINGER.COM/ARTICLE/10.1007/S12152016-9295-2. [Fecha de consulta: 13.1.2020].

PicKard, Hanna, 2018: "The Puzzle of Addiction”, en Hanna Pickard y Serge Ahmed (editores), The Routledge Handbook of Philosopby and Sciencie of Addiction, Londres, Routledge, pp. 9-22. Disponible en: HTTPS://WWW.ROUTLEDGEHANDBOOKS.COM/ DOI/10.4324/9781315689197. [Fecha de consulta: 13.1.2020].

Sedgewick, Peter, 1982: Psycho Politics: Laing, Foucault, Goffman, Szasz, and the Future of Mass Psychiatry, New York: Harper \& Row.

SCUlLY, Jackie, 2013: "Disability and vulnerability: On bodies, dependence and power", Wendy Rogers, Catriona Mackenzie y Susan Dodds (editoras), Vulnerability: New essays in ethics and feminist philosophy, Oxford, Oxford University press, pp. 204-221.

Szlemko, W., Wood, J., y Thurman, P., 2006: "Native Americans and Alcohol: Past, Present, and Future", The Journal of General Psychology, Vol. 133, № 4. Disponible en HTTPS:// WWW.TANDFONLINE.COM/DOI/ABS/10.3200/GENP. 133.4.435-451. [Fecha de consulta: 11.6.2020].

Teeson, M., Hall, W., Proudfoot, H., y Degendhardt, L., 2012: Addictions (2a edición), New York, Psychology Press.

Universidad de Antioquia y Organización Indígena de Antioquia (2006). Estado del arte del conocimiento sobre la salud de los pueblos indígenas de América: Salud mental de los pueblos indígenas. Recuperado el 6.1.2020, de Universidad de Antioquia Salud Pública Virtual: HTTP://SALUDPUBLICAVIRTUAL.UDEA.EDU.CO/CVSP/LA\%20SALUD\%20 INDIGENA.PDF.

Vargas-Espíndola, A., Villamizar-Guerrero, J., Puerto-López, J., Rojas-Villamizar, M., Ramírez-Montes, O., Urrego-Mendoza, Z., 2017: "Conducta suicida en pueblos indígenas: una revisión del estado del arte”, Rev. Fac. Med., volumen 65, No 1 . Disponible en HTTP://WWW.SCIELO.ORG.CO/SCIELO.PHP?SCRIPT=SCI_ABSTRACT\&PID =S0120-00112017000100129. [Fecha de consulta: 6.1.2020].

WAKefield, Jerome y Schmitz, Mark, 2015: "How many people have alcohol use disorders? Using the harmful dysfunction analysis to reconcile prevalence estimates in two community surveys", en Hanna Pickard, Serge Ahmed y Bennett Foddy (editores), Alternative Models of Addiction, Lausanne, Frontiers in Psychiatry, pp. 89-110. Disponible en: HTTPS://WWW. FRONTIERSIN.ORG/RESEARCH-TOPICS/1036/ALTERNATIVE-MODELS-OF-ADDICTION. [Fecha de consulta: 13.1.2020].

Wallcraft, Jan y Hopper, Kim, 2015: "The Capabilities Approach and the social model of mental health", en Helen Splandler, Jill Anderson y Bob Sapey (editores), Madness, Distress and the Politics of Disablement, Bristol, The Policy Press, pp. 83-97.

Wasserman, David, 2004: "Addiction and Disability: Moral and Policy Issues", Substance Use E Misuse, volumen 39, $\mathrm{N}^{\circ}$ 3. Disponible en HTTPS://WWW.TANDFONLINE.COM/DOI/ ABS/10.1081/JA-120029986? JOURNALCODE=ISUM20. [Fecha de consulta: 13.2.2020]. 
Wesley-Esquimaux, Cynthia y Smolewski, Magdalena, 2004: Historic Trauma and Aboriginal Healing, Ottawa: Aboriginal Healing Foundation. Disponible en HTTP://WWW.AHF.CA/ DOWNLOADS/HISTORIC-TRAUMA.PDF. [Fecha de consulta: 11.6.2020].

\section{Normas jurídicas citadas}

Convención sobre la Eliminación de todas las Formas de Discriminación Racial de 1965. Aprobada en Colombia mediante Ley $\mathrm{N}^{\circ} 22$ de 1981.

Ley 30 de 1986, Congreso de la República de Colombia.

Convenio No 169 sobre Pueblos Indígenas y Tribales de 1989. Aprobada en Colombia mediante Ley $\mathrm{N}^{\circ} 21$ de 1991.

Decreto 1811 de 1990, Presidencia de la República de Colombia.

Resolución 46/119 de 1991, Asamblea General de la ONU.

Ley 691 de 2001, Congreso de la República de Colombia.

Resolución 61/295 de 2007, Asamblea General de la ONU.

Acto legislativo 02 de 2009, Congreso de la República de Colombia.

Convención sobre los Derechos de las Personas con Discapacidad. Aprobada en Colombia mediante Ley $\mathrm{N}^{\circ} 1346$ de 2009.

LEy N 1438 de 2011, Congreso de la República de Colombia.

LEY N 1566 de 2012, Congreso de la República de Colombia.

LEY N 1616 de 2013, Congreso de la República de Colombia.

Ley Estatutaria 1751 de 2015, Congreso de la República de Colombia.

\section{Jurisprudencia citada}

Corte Constitucional de Colombia, sentencia de 22 de agosto de 2002, T-684/02.

Corte Constitucional de Colombia, auto de fecha 26 de enero de 2009, Auto 004 de 2009.

Corte Constitucional de Colombia, sentencia de 3 de julio de 2009, T-438/09.

Corte Constitucional de Colombia, sentencia de 9 de febrero de 2012, T-057/12.

Corte Constitucional de Colombia, sentencia de 7 de julio de 2014, T-454/14.

Corte Constitucional de Colombia, sentencia de 4 de febrero de 2015, T-043/15.

Corte Constitucional de Colombia, sentencia de 22 de mayo de 2015, T-318/15.

Tribunal Superior del Distrito Judicial de Arauca, sentencia de $1^{\circ}$ de julio de 2015 , Rad. 2014-00052-03.

Corte Constitucional de Colombia, sentencia de 7 de julio de 2015, T-418/15.

Contraloría General de la República de Colombia, informe de junio de 2016. Resultado actuación especial de seguimiento al fallo de tutela de radicado 2014-00052-03.

Corte Constitucional de Colombia, sentencia de 16 de septiembre de 2016, T-511/16.

Corte Constitucional de Colombia, sentencia de 22 de enero de 2019, T-011/19. 